\title{
A Matrix-Based Approach to the Image Moment Problem
}

\author{
JUDIT MARTINEZ, JOSEP M. PORTA AND FEDERICO THOMAS \\ Insitut de Robòtica i Informàtica Industrial (CSIC-UPC), Llorens Artigas 4-6, 08028 Barcelona, Spain \\ \{porta,thomas\}@iri.upc.edu
}

\begin{abstract}
An image can be seen as an element of a vector space and hence it can be expressed in as a linear combination of the elements of any non necessarily orthogonal basis of this space. After giving a matrix formulation of this well-known fact, this paper presents a reconstruction method of an image from its moments that sheds new light on this inverse problem. Two main contributions are presented: (a) the results using the standard approach based on the least squares approximation of the result using orthogonal polynomials can also be obtained using matrix pseudoinverses, which implies higher control on the numerical stability of the problem; and (b) it is possible to use basis functions in the reconstruction different from orthogonal polynomials, such as Fourier or Haar basis, allowing to introduce constraints relative to the bandwidth or the spatial resolution on the image to be reconstructed.
\end{abstract}

Keywords: The moment problem, image moments, moment-invariant image approximations, basis selection.

\section{Introduction}

A very common problem in physics and engineering is known under the general title of "the moment problem" [18]. Corresponding to some finite number of observations, we are given a set of moments -the integrals of various given functions with respect to the measure. Since these moments will not determine the measure uniquely, the problem consists in deciding which is the best estimate. In pure mathematics, this problem dates back to Theodor Stieltjes who proposed it in a paper published in 1894. His work on the moment problem was continued and extended primarily by Hausdorff and Hamburger. For classical overviews of the subject, including comprehensive historical attributions of classical results, see [1] or [16]. A more recent survey of the wide range of approaches to the problem, including applications, is [5].

This paper is concerned with the moment problem for images or, more precisely, with the problem of reconstructing an image from a set of its geometric moments.

The moment problem for images arises in several applications. In [11], the problem of inverting the Radon transform is reformulated into that of reconstructing an image from estimates of its moments. In [13], the moment problem has also arisen when approximating an image to simplify it. In this work, a finite number of moments are used to reconstruct an approximation of the Fourier coefficients of the corresponding image. Unfortunately, images are not treated as 2D discrete functions. Instead, using a zigzag scan, they are converted into a linear form.

The reconstruction of an image from a set of its moments is not necessarily unique. In other words, it is an ill-posed problem. Therefore, all possible methods to solve it must impose extra constraints so that the solution becomes unique.

The standard reconstruction method of an image from some of its moments is based on the leastsquares approximation of the image using orthogonal polynomials $[17,15,12]$. Polynomials are the most straightforward choice among all possible orthogonal basis functions because they can be easily related to the multinomial functions that are used to obtain the geometric moments. Legendre and Zernike polynomials were first used in [17]. They are orthogonal polynomials for continuous variables in rectangular and polar coordinates, respectively. However, they are not orthogonal for 
discrete variables, contrary to what is assumed by some authors $[15,6]$. Tchebichef polynomials were used in [8] and [12] which are orthogonal polynomials in the discrete domain. Independently of the chosen set of polynomials, the standard method assumes null projection coefficients onto the chosen polynomial set of order higher than the maximum order of available moments. This solves the ill-posesness and the solution becomes unique. In order to avoid this assumption, which is difficult to interpret in terms of the image properties, a maximum entropy method was proposed in [14]. It consists in obtaining the image with maximum entropy with the desired moments. Solving the problem using Lagrange multipliers permits to obtain an explicit form of the reconstructed image in terms of an exponential function. Alternatively, [10] proposes minimizing the divergence of the image, instead of maximizing its entropy, using also a variational approach. Unfortunately, both approaches assume a continuous domain for the image.

In this paper, we propose a reconstruction method that permits introducing constraints that can be interpreted in terms of image properties, such as bandwidth or spatial resolution. We also show how the standard least-squares reconstruction method can be seen as a particular case of it. First, we introduce the necessary mathematical background. Section 3. reformulates the standard method in terms of the presented formalism. Section 4. generalizes the result to other orthogonal bases different from polynomials. Finally, section 5. contains the conclusions and prospects for future research.

\section{Mathematical background}

\subsection{Notation and definitions}

Let $\mathbf{z}_{m} \in \Re^{m}$ denote a column vector, $\mathbf{z}_{m}^{t}$ its transpose, and $\mathbf{z}_{m}[k]$, with $k=1, \ldots, m$, each of its elements. Likewise, let $\mathbf{Z}_{m n} \in \Re^{m \times n}$ denote a matrix of size $m \times n$ and $\mathbf{Z}_{m n}[k, l]$, its element $(k, l)$, where $k=1, \ldots, m$ and $l=1, \ldots, n$. For simplicity, square matrices will only have one subscript. Superscripts are used to denote any parameter on which a matrix depends. Two unary matrix operations are used: $(\cdot)^{t}$ denotes the transpose of a given matrix; and $(\cdot)^{-1}$, its inverse. To avoid confusions, matrices are always embraced by parenthesis when superscripts refer to power or transpose.

Any discrete image of size $a \times b$, say $\mathbf{I}_{a b}$, can be seen as a vector in $\Re^{a \times b}$ or, alternatively, as a bidimensional function that maps all the points of the uniform lattice $\{1,2, \ldots, a\} \times\{1,2, \ldots, b\}$ onto real values. Then, $\mathbf{I}_{a b}$ can be uniquely expressed as a linear combination of the functions of a basis set, i.e., a set containing $a b$ linearly independent bidimensional functions, which will be denoted by the set $\left\{\boldsymbol{\Xi}_{a b}^{k l}\right\}$, so that

$$
\mathbf{I}_{a b}=\sum_{k=1}^{a} \sum_{l=1}^{b} \alpha^{k l} \boldsymbol{\Xi}_{a b}^{k l}
$$

To avoid in what follows this double summation in the formulation of the problem, we introduce a matrix-based formulation, but first we need some definitions.

Definition 1 (Basis matrix). The functions in any basis set are assumed to be separable and equally defined for both coordinates, i.e., $\boldsymbol{\Xi}_{a b}^{k l}=$ $\phi_{a}^{k}\left(\phi_{b}^{l}\right)^{t}$, where $\phi_{a}^{k}$ and $\phi_{b}^{l}$ are vectors which will be grouped in matrices of the form $\boldsymbol{\Phi}_{a b}=$ $\left(\phi_{a}^{1}, \ldots, \phi_{a}^{b}\right)$ called basis matrices.

Definition 2 (Gram matrix). The matrix $\boldsymbol{\Gamma}_{b}^{a}=$ $\left(\boldsymbol{\Phi}_{a b}\right)^{t} \boldsymbol{\Phi}_{a b}$, containing the inner products between the elements of the corresponding basis matrix, is called a Gram matrix.

Note that, since $\boldsymbol{\Gamma}_{b}^{a}[k, l]=\left\langle\boldsymbol{\phi}_{a}^{k}, \boldsymbol{\phi}_{a}^{l}\right\rangle$, the Gram matrices are diagonal for orthogonal basis sets and the identity for orthonormalized basis.

Definition 3 (Projection matrix). The matrix containing the projection coefficients of image $\mathbf{I}_{a b}$ onto the first $m \times n$ elements of $\left\{\boldsymbol{\Xi}_{a b}^{k l}\right\}$ are called projection matrices, which can be expressed as $\boldsymbol{\Omega}_{m n}=\left(\boldsymbol{\Phi}_{a m}\right)^{t} \mathbf{I}_{a b} \boldsymbol{\Phi}_{b n}$.

Note that $\boldsymbol{\Omega}_{m n}[k, l]=\left\langle\mathbf{I}_{a b}, \boldsymbol{\Xi}_{a b}^{k l}\right\rangle=\left(\boldsymbol{\phi}_{a}^{k}\right)^{t} \mathbf{I}_{a b} \boldsymbol{\phi}_{b}^{l}$.

Image $\mathbf{I}_{a b}$ can be approximated in terms of the first $m \times n$ elements of $\left\{\boldsymbol{\Xi}_{a b}^{k l}\right\}$ by

$$
\hat{\mathbf{I}}_{a b}^{m n}=\sum_{k=1}^{m} \sum_{l=1}^{n} \lambda^{k l} \boldsymbol{\Xi}_{a b}^{k l}=\boldsymbol{\Phi}_{a m} \boldsymbol{\Lambda}_{m n}\left(\boldsymbol{\Phi}_{b n}\right)^{t},
$$

where $m \leq a, n \leq b$, and $\boldsymbol{\Lambda}_{m n}[k, l]=\lambda^{k l}$. 
Definition 4 (Expansion matrix). If the image approximation coefficients $\lambda^{k l}$ are chosen so that the truncation error is minimized using the leastsquares error criterion, $\boldsymbol{\Lambda}_{m n}$ is called an expansion matrix.

\subsection{A Theorem}

Lemma 1. The approximation of image $\mathbf{I}_{a b}$, in the least-squares sense, can be expressed in terms of the projection matrix $\boldsymbol{\Omega}_{m n}$ as

$$
\begin{aligned}
\hat{\mathbf{I}}_{a b}^{m n}= & \boldsymbol{\Phi}_{a m} \boldsymbol{\Lambda}_{m n}\left(\boldsymbol{\Phi}_{b n}\right)^{t} \\
= & \boldsymbol{\Phi}_{a m}\left(\boldsymbol{\Gamma}_{m}^{a}\right)^{-1} \boldsymbol{\Omega}_{m n}\left(\boldsymbol{\Gamma}_{n}^{b}\right)^{-1}\left(\boldsymbol{\Phi}_{b n}\right)^{t} \\
= & \boldsymbol{\Phi}_{a m}\left(\left(\boldsymbol{\Phi}_{a m}\right)^{t} \boldsymbol{\Phi}_{a m}\right)^{-1} \\
& \quad \boldsymbol{\Omega}_{m n}\left(\left(\boldsymbol{\Phi}_{b n}\right)^{t} \boldsymbol{\Phi}_{b n}\right)^{-1}\left(\boldsymbol{\Phi}_{b n}\right)^{t} \\
& =\left(\boldsymbol{\Phi}_{a m}\right)^{-} \boldsymbol{\Omega}_{m n}\left(\boldsymbol{\Phi}_{b n}\right)^{+},
\end{aligned}
$$

where $(\cdot)^{-}$and $(\cdot)^{+}$stand for the left and right Moore-Penrose pseudoinverses.

Proof. Since $\lambda^{k l}$ is chosen so that the truncation error is minimized according to the least-squares error criterion, the subspaces generated by the error and that in which the approximated image is contained are orthogonal. That is,

$$
\left\langle\boldsymbol{\Xi}_{a b}^{i j}, \mathbf{I}_{a b}-\boldsymbol{\Phi}_{a m} \boldsymbol{\Lambda}_{m n}\left(\boldsymbol{\Phi}_{b n}\right)^{t}\right\rangle=0,
$$

for $i=1, \ldots, m$ and $j=1, \ldots, n$. Then,

$$
\left\langle\boldsymbol{\Xi}_{a b}^{i j}, \mathbf{I}_{a b}\right\rangle=\left\langle\boldsymbol{\Xi}_{a b}^{i j}, \boldsymbol{\Phi}_{a m} \boldsymbol{\Lambda}_{m n}\left(\boldsymbol{\Phi}_{b n}\right)^{t}\right\rangle .
$$

Hence, when translating these $m \times n$ scalar equations into a single matrix equation, we get

$\boldsymbol{\Omega}_{m n}=\left(\boldsymbol{\Phi}_{a m}\right)^{t} \boldsymbol{\Phi}_{a m} \boldsymbol{\Lambda}_{m n}\left(\boldsymbol{\Phi}_{b n}\right)^{t} \boldsymbol{\Phi}_{b n}=\boldsymbol{\Gamma}_{m}^{a} \boldsymbol{\Lambda}_{m n} \boldsymbol{\Gamma}_{n}^{b}$.

Since Gram matrices are obtained from functions of a basis set, they are non-singular and this proves the Lemma.

Corollary 1. If the basis set $\left\{\overline{\boldsymbol{\Xi}}_{a b}^{k l}\right\}$ is orthonormal - we use an overline to distinguish it from the general case - the least-squares approximation of the image can be expressed as

$$
\overline{\mathbf{I}}_{a b}^{m n}=\overline{\mathbf{\Phi}}_{a m} \overline{\mathbf{\Omega}}_{m n}\left(\overline{\mathbf{\Phi}}_{b n}\right)^{t},
$$

because $\overline{\boldsymbol{\Omega}}_{m n}=\overline{\boldsymbol{\Lambda}}_{m n}$.

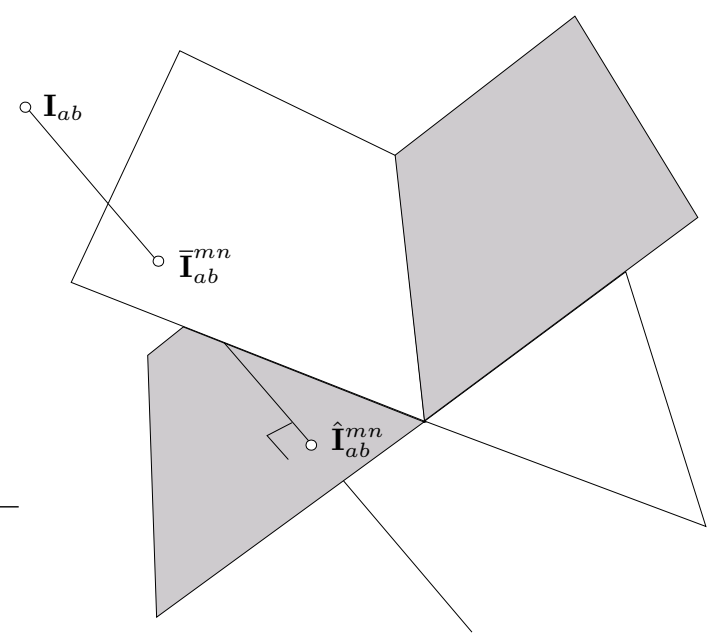

Figure 1: Lemma 1 permits to obtain the best approximation, $\hat{\mathbf{I}}_{a b}^{m n}$, of image $\mathbf{I}_{a b}$ in the least-squares sense, contained in the subspace represented by the plane in gray, that is, its orthogonal projection onto this subspace. Theorem 1 is a generalization of this lemma that permits to obtain the image $\overline{\mathbf{I}}_{a b}^{m n}$ contained in other subspaces, here represented by a white plane, that also projects orthogonally onto $\hat{\mathbf{I}}_{a b}^{m n}$. Both $\overline{\mathbf{I}}_{a b}^{m n}$ and $\hat{\mathbf{I}}_{a b}^{m n}$ preserve the first $m \times n$ moments of $\mathbf{I}_{a b}$.

There are infinite images, not only $\mathbf{I}_{a b}$, that lead to the same projection matrix, $\boldsymbol{\Omega}_{m n}$, resulting from projecting them onto the first $m \times n$ elements of the basis $\left\{\boldsymbol{\Xi}_{a b}^{k l}\right\}$. The above lemma permits to choose from this infinite set the one contained in the subspace spanned by the first $m \times n$ elements of the basis $\left\{\boldsymbol{\Xi}_{a b}^{k l}\right\}$. The following theorem allows us to select other images contained in spaces spanned by other arbitrary orthogonal basis, $\left\{\overline{\boldsymbol{\Xi}}_{a b}^{k l}\right\}$, whose first $m \times n$ elements not necessarily span the same subspace as $\left\{\boldsymbol{\Xi}_{a b}^{k l}\right\}$. Fig. 1 gives a geometric interpretation of this fact.

Theorem 1. Given the projection matrix $\boldsymbol{\Omega}_{m n}=$ $\left(\boldsymbol{\Phi}_{a m}\right)^{t} \mathbf{I}_{a b} \boldsymbol{\Phi}_{b n}$, the image contained in the subspace expanded by an orthonormal basis, with basis matrix $\overline{\mathbf{\Phi}}_{\text {am }}$, which leads to the same projection matrix $\boldsymbol{\Omega}_{m n}$, is given by

$$
\begin{aligned}
\overline{\mathbf{I}}_{a b}^{m n}= & \overline{\boldsymbol{\Phi}}_{a m}\left(\left(\boldsymbol{\Phi}_{a m}\right)^{t} \overline{\boldsymbol{\Phi}}_{a m}\right)^{-1} \\
& \boldsymbol{\Omega}_{m n}\left(\left(\overline{\boldsymbol{\Phi}}_{b n}\right)^{t} \boldsymbol{\Phi}_{b n}\right)^{-1}\left(\overline{\boldsymbol{\Phi}}_{b n}\right)^{t} \\
= & \overline{\boldsymbol{\Phi}}_{a m}\left(\mathbf{C}_{m}^{a}\right)^{-1} \boldsymbol{\Omega}_{m n}\left(\left(\mathbf{C}_{n}^{b}\right)^{t}\right)^{-1}\left(\overline{\mathbf{\Phi}}_{b n}\right)^{t}
\end{aligned}
$$

where $\mathbf{C}_{q}^{p}[k, l]=\left\langle\overline{\phi_{p}^{k}}, \phi_{p}^{l}\right\rangle$. 
Proof. Let us consider the following approximated image

$$
\overline{\mathbf{I}}_{a b}^{m n}=\overline{\mathbf{\Phi}}_{a m} \overline{\mathbf{\Omega}}_{m n}\left(\overline{\mathbf{\Phi}}_{b n}\right)^{t} .
$$

If we want this image to have the same projection coefficients onto $\left\{\boldsymbol{\Xi}_{a b}^{k l}\right\}$ as $\mathbf{I}_{a b}$, then

$$
\begin{aligned}
\boldsymbol{\Omega}_{m n} & =\left(\boldsymbol{\Phi}_{a m}\right)^{t} \mathbf{I}_{a b} \boldsymbol{\Phi}_{b n}=\left(\boldsymbol{\Phi}_{a m}\right)^{t} \overline{\mathbf{I}}_{a b}^{m n} \boldsymbol{\Phi}_{b n} \\
& =\left(\boldsymbol{\Phi}_{a m}\right)^{t} \overline{\boldsymbol{\Phi}}_{a m} \overline{\boldsymbol{\Omega}}_{m n}\left(\overline{\boldsymbol{\Phi}}_{b n}\right)^{t} \boldsymbol{\Phi}_{b n} .
\end{aligned}
$$

Thus, if $\left(\boldsymbol{\Phi}_{a m}\right)^{t} \overline{\boldsymbol{\Phi}}_{a m}$ and $\left(\overline{\mathbf{\Phi}}_{b n}\right)^{t} \boldsymbol{\Phi}_{b n}$ are non singular, we have that

$$
\overline{\boldsymbol{\Omega}}_{m n}=\left(\left(\boldsymbol{\Phi}_{a m}\right)^{t} \overline{\boldsymbol{\Phi}}_{a m}\right)^{-1} \boldsymbol{\Omega}_{m n}\left(\left(\overline{\boldsymbol{\Phi}}_{b n}\right)^{t} \boldsymbol{\Phi}_{b n}\right)^{-1},
$$

which, when substituted in equation (1), proves the lemma.

Now, the least-squares approximation given by Lemma 1 can be seen as the particular case of this theorem in which the used orthogonal basis expands the same subspace as the basis used in the projection.

Before describing the applications of the above theorem, let us reformulate the least-squares method in terms of the matrix formalism just introduced.

\section{Revisiting the standard method}

We define the centered geometric moment of order $(k, l)$ of image $\mathbf{I}_{a b}$ as

$$
\mu^{k l}=\sum_{x=1}^{a} \sum_{y=1}^{b}(x-\lfloor a / 2\rfloor)^{k}(y-\lfloor b / 2\rfloor)^{l} \mathbf{I}_{a b}[x, y] .
$$

Then, $\mu^{k l}$ can be seen as a projection coefficient of the image onto a multinomial basis, and the first $m \times n$ moments of $\mathbf{I}_{a b}$ can be expressed in matrix form as (see Lemma 1 in [9] for details):

$$
\begin{aligned}
\boldsymbol{\Omega}_{m n} & =\left(\boldsymbol{\Phi}_{a m}\right)^{t} \mathbf{I}_{a b} \boldsymbol{\Phi}_{b n} \\
& =\left(\mathbf{T}_{m}\right)^{t}\left(\mathbf{V}_{a m}\right)^{t} \mathbf{I}_{a b} \mathbf{V}_{b n} \mathbf{T}_{n},
\end{aligned}
$$

where

$$
\begin{gathered}
\boldsymbol{\Omega}_{m n}[k, l]=\mu^{k-1 l-1}, \\
\mathbf{T}_{p}[k, l]= \begin{cases}\left(\begin{array}{l}
l-1 \\
k-1
\end{array}\right)(-\lfloor p / 2\rfloor)^{l-k}, & \text { if } l \geq k, \\
0, & \text { otherwise }\end{cases}
\end{gathered}
$$
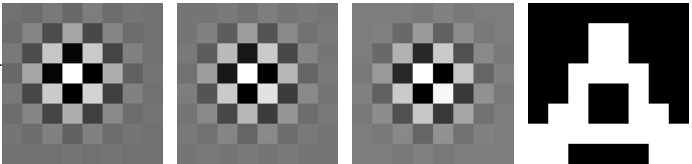

$m=n=2$

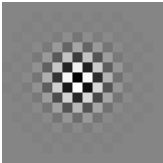

$m=n=4$
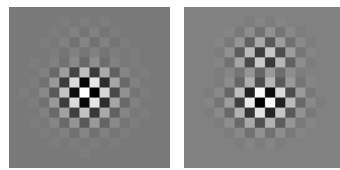

$m=n=8$

$m=n=4 \quad m=n=8 \quad m=n=12 m=n=16$

Figure 2: Reconstruction of a $8 \times 8$ pattern (top) and a $16 \times 16$ pattern (bottom) by imposing null values to the unknown moments. Double precision used.

and $\mathbf{V}_{p q}$ is a non-square Vandermode matrix whose general term is:

$$
\mathbf{V}_{p q}[k, l]=k^{l-1}
$$

In most applications involving moments, the idea is to use the lowest number of moments as possible, that is, $\max (m, n) \ll \min (a, b)$. As a consequence, the matrices $\boldsymbol{\Phi}_{a m}$ and $\boldsymbol{\Phi}_{b n}$ are seldom square and, given $\boldsymbol{\Omega}_{m n}$, there are infinite solutions for $\mathbf{I}_{a b}$ satisfying (2).

\subsection{A naive approach}

We can devise a naive reconstruction method by simply assuming that all unknown moments ranging from order $(m, n)$ to order $(a, b)$ are zero. Then, let us define

$$
\tilde{\boldsymbol{\Omega}}_{a b}=\left(\begin{array}{ll}
\boldsymbol{\Omega}_{m n} & \mathbf{0}_{m(b-n)} \\
\mathbf{0}_{(a-m) n} & \mathbf{0}_{(a-m)(b-n)}
\end{array}\right) .
$$

According to (2), the image with the moments given by (6) is:

$$
\tilde{\mathbf{I}}_{a b}=\left(\left(\boldsymbol{\Phi}_{a a}\right)^{t}\right)^{-1} \tilde{\boldsymbol{\Omega}}_{a b}\left(\boldsymbol{\Phi}_{b b}\right)^{-1} .
$$

Unfortunately, although the image thus obtained preserves the desired moments up to order $(m, n)$, in practice the result has little relation with the original image. Indeed, imposing zero values to unknown moments leads, in general, to images with negative pixel values and large variations. In practice, when the dimensions of the original pattern and the projection matrix do not coincide, the result does not resembles the original. This 


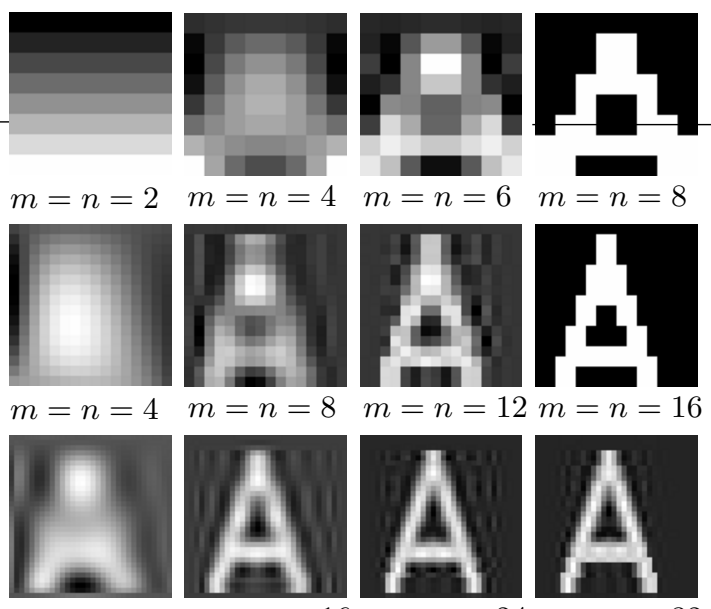

$m=n=8 \quad m=n=16 m=n=24 m=n=32$

Figure 3: Reconstructing a $8 \times 8$ pattern (top), a $16 \times 16$ pattern (middle), and a $32 \times 32$ pattern (bottom) from its moments using the approximation derived from Lemma 1.

phenomenon is exemplified for a $8 \times 8$ pattern in Fig. 2-top. If we increase the size of the pattern (see Fig. 2-bottom), the original image cannot be reconstructed even in the case that the size of the projection matrix and the image coincide. To understand what is the problem, it is enough to realize that the factorization of $\left(\boldsymbol{\Phi}_{a a}\right)^{t}$ involves a Vandermonde matrix (see equation 2). This matrix is extremely ill-conditioned, so that standard numerically stable methods in general fail to compute its inverse accurately, even for moderate sizes. In fact, its condition number grows exponentially with its size [2] so that, using a double precision representation, this matrix cannot be properly inverted using a standard inversion algorithm. This is what happened in the example presented in Fig. 2-bottom.

In sum, this naive approach has little practical interest but it has been useful to surface an important problem: numerical conditioning.

\subsection{Using Lemma 1}

Using Lemma 1, we have

$$
\hat{\mathbf{I}}_{a b}^{m n}=\boldsymbol{\Phi}_{a m}\left(\boldsymbol{\Gamma}_{m}^{a}\right)^{-1} \boldsymbol{\Omega}_{m n}\left(\boldsymbol{\Gamma}_{n}^{b}\right)^{-1}\left(\boldsymbol{\Phi}_{b n}\right)^{t}
$$

where the Gram matrix $\boldsymbol{\Gamma}_{m}^{a}=\left(\boldsymbol{\Phi}_{a m}\right)^{t} \boldsymbol{\Phi}_{a m}$ is squared and can be directly inverted. Now, the image approximation is obtained inverting two Gram
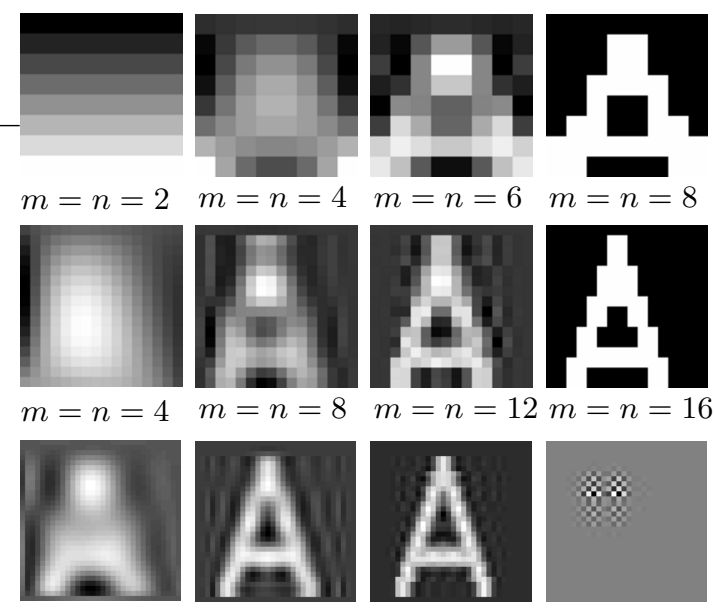

$m=n=4 \quad m=n=6 \quad m=n=8$

$m=n=8$
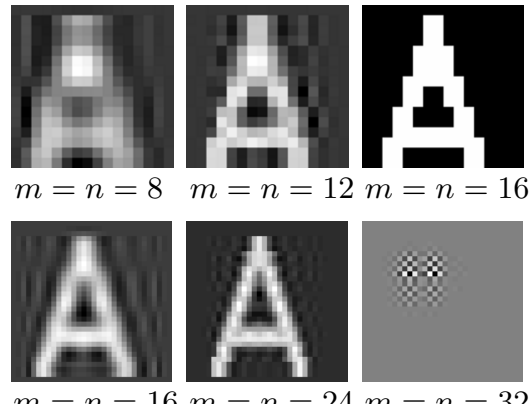

Figure 4: Reconstructing a $8 \times 8$ pattern (top) and a $16 \times 16$ pattern (middle), and a $32 \times 32$ pattern (bottom) from its moments using the approximation derived from Theorem 1 and the Tchebichev basis.

matrices of size $m \times m$ and $n \times n$, independently of the size of the image.

Fig. 3 presents some examples using IEEE 754 double float representation and Gauss elimination inversion.

In Fig. 3 -top, the reconstruction of a $8 \times 8$ pattern is carried out using equation (7). It can be seen how the result converges as the order of used moments increases.

Fig. 3-middle and 3-bottom show the reconstruction of a $16 \times 16$ and $32 \times 32$ pattern, respectively. The sizes of the inverted Gram matrices range from $4 \times 4$ to $32 \times 32$. When the size of the image and the moment matrix coincide, the reconstructed image and the original pattern should coincide but, due again to numerical instabilities, it is not so.

Table. 1 shows the maximum size of the projection matrices so that the the mean quadratic error between the identity matrix and $\left(\boldsymbol{\Gamma}_{m}^{a}\right)^{-1} \boldsymbol{\Gamma}_{m}^{a}$, using IEEE double float representation and Gauss elimination inversion, is lower that 0.0001 . Images with sizes equal or lower than those maxima can be safely reconstructed without resorting to more sophisticated inversion algorithms better suited for ill-conditioned systems [4]. 


\begin{tabular}{c|c} 
Reconstruction Method & $m$ \\
\hline Lemma 1 & 12 \\
Theorem 1 + Tchebichev & 16 \\
Theorem 1 + Fourier & 15 \\
Theorem 1 + Haar & 8
\end{tabular}

Table 1: Size of the projection matrices, $m$, for which negligible reconstruction error are observed for different reconstruction methods. In each case, images of size $a \times a$, with $a \leq m$, can be reconstructed with a mean quadratic error lower than 0.0001. These results are obtained using Gauss elimination inversion and IEEE 754 double precision representation. Note that the Haar basis is only defined for $m$ power of 2 .

\subsection{Using Theorem 1}

In Fig. 4, the reconstruction of the same binary pattern as above is carried out using Lemma 2 and taking as basis $\left\{\overline{\boldsymbol{\Xi}}_{a b}^{k l}\right\}$ the normalized Tchebichef polynomials [12], so that the corresponding basis matrix is

$$
\boldsymbol{\Phi}_{p q}[k, l]=\frac{\mathbf{t}_{p}^{l}[k]}{\sqrt{(2 l-2) ! c(p+l-1,2 l-1)}},
$$

where

$$
\begin{array}{r}
\mathbf{t}_{p}^{l}[k]=(l-1) ! \sum_{j=1}^{l}(-1)^{l-j} c(p-j, l-j) \\
c(l-2+j, l-1) c(k-1, j-1),
\end{array}
$$

and $c(a, b)$ is the generalization of the binomial numbers given by

$$
c(j, k)=\left\{\begin{array}{lll}
1 & \text { if } & k=0 \\
\left(\begin{array}{l}
j \\
k
\end{array}\right) & \text { if } & 0<k \leq j, \\
0 & \text { if } & k>j .
\end{array}\right.
$$

Note how numerical problems also arise. As in the previous example, Table. 1 indicate the image sizes for which the reconstruction can safely done using IEEE double float representation and Gauss elimination inversion.

Since the Tchebichef polynomials and the moments basis expand the same subspace, the Corollary 2 ensures that the reconstructed image using this approximation and that obtained via Lemma 1 are the same (up to numerical instabilities). Thus, it can be concluded that there is not

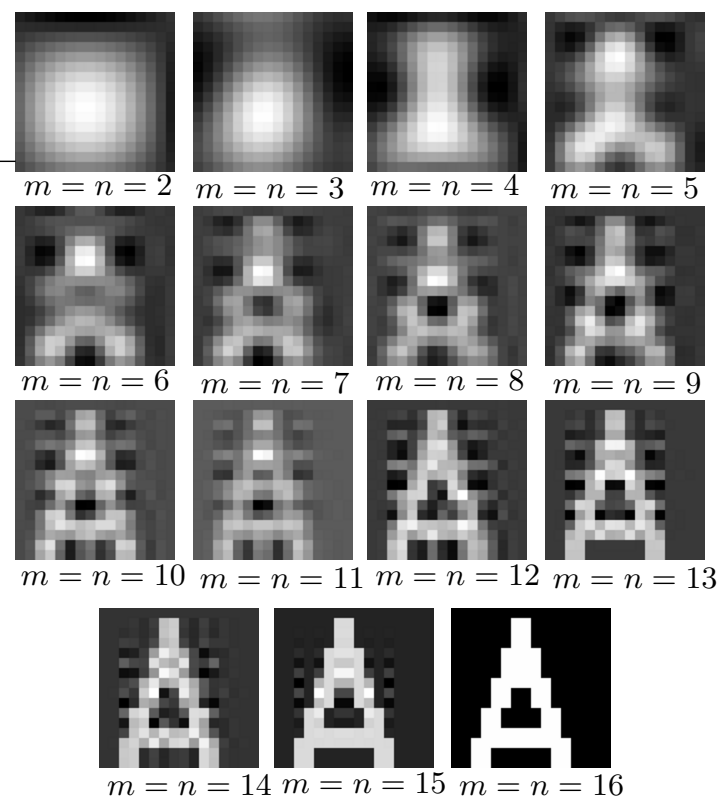

Figure 5: Reconstructing a $16 \times 16$ pattern from its moments using the approximation derived from Theorem 1 and the Fourier basis.

necessary to use an orthogonal basis, contrary to what has been assumed in the literature, because the use the Lemma 1 based reconstruction method yields the same practical results.

\section{A Novel Reconstruction Method}

In this section, we explore the possibility that the projection and the reconstruction subspaces are not the same by applying Lemma 2 .

\subsection{Reconstructing a band-limited image}

While derivatives give information on the high frequencies of a signal, moments give information on its low frequencies. This duality is clear by realizing that a one-dimensional real function $f(t)$ can be expressed in terms of its Maclaurin expansion as

$f(t)=f(0)+f^{\prime}(0) t+\frac{f^{\prime \prime}(0)}{2 !} t^{2}+\cdots+\frac{f^{(n)}(0)}{n !} t^{n}+\ldots$

and its Fourier transform, say $F(w)$, as

$F(w)=m_{0}-j w m_{1}+\frac{(j w)^{2}}{2} m_{2}+\cdots+\frac{(-1)^{n}(j w)^{n}}{n !} m_{n}+\ldots$ 
where $m_{i}$ the moment of order $i$ of $f(x)$.

Next, the relationship between moments and low frequencies is made explicit for discrete images.

Fourier coefficients are normally defined as

$f^{k l}=\frac{1}{\sqrt{a b}} \sum_{x=1}^{a} \sum_{y=1}^{b} \mathbf{I}_{a b} \mathrm{e}^{-j 2 \pi\left(\frac{(x-1)(k-1)}{a}+\frac{(y-1)(l-1)}{b}\right)}$.

A relocation of these coefficients in a matrix can be carried out so that increasing indexes correspond to higher frequency coefficients as follows:

$$
\begin{aligned}
& \mathbf{F}_{m n}[k, l]=\frac{1}{\sqrt{a b}} \sum_{x=1}^{a} \sum_{y=1}^{b} \mathbf{I}_{a b} \\
& \mathrm{e}^{-j 2 \pi\left(\frac{(x-1)\left(k-\frac{(m-1)}{2}-1\right)}{a}+\frac{\left(y-\frac{(n-1)}{2}-1\right)(l-1)}{b}\right)} .
\end{aligned}
$$

Then, Fourier coefficients can be seen as the projection coefficients of the image onto a set of complex exponential basis functions that lead to the basis matrix

$$
\overline{\mathbf{\Phi}}_{p q}[k, l]=\frac{1}{\sqrt{p}} \mathrm{e}^{-j 2 \pi\left(\frac{(k-1)\left(l-\frac{(q-1)}{2}-1\right)}{p}\right)} .
$$

Substituting these orthogonal basis matrices in the result of Lemma 2, a low-pass approximation of the original image is obtained from a subset of its geometric moments. Fig. 5 shows the obtained results using the same pattern as in the previous examples. As above, Table 1 indicate the image sizes for which the reconstruction can be safely done.

\subsection{Reconstructing a resolution-limited image}

Limiting the resolution of an image means eliminating those regions of smaller size than a given one. In terms of the Haar transform, this requirement becomes trivial since its main characteristic is the direct relationship between the number of coefficients and the spatial resolution of the image.

Haar coefficients are obtained from the projection of the image onto the discrete Haar functions $\mathbf{h}_{p}^{l}[k]$, for $p$ a power of 2 , defined for $l=1$ as

$$
\mathbf{h}_{p}^{1}[k]=\frac{1}{\sqrt{p}}
$$

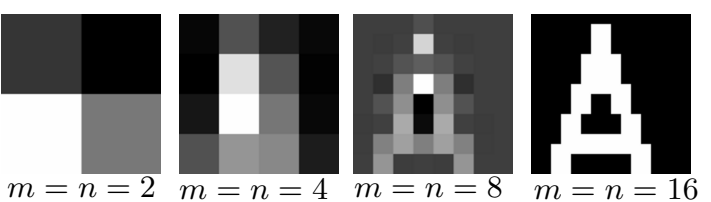

Figure 6: Reconstructing a $16 \times 16$ pattern from its moments using the approximation derived from Theorem 1 and the Haar basis.

and for $l>1$ as

$$
\mathbf{h}_{p}^{l}[k]= \begin{cases}+\sqrt{\frac{r}{p}} & \text { if } \quad s \leq k<s+\frac{p}{2 r} \\ -\sqrt{\frac{r}{p}} & \text { if } \quad s+\frac{p}{2 r} \leq k \leq s+\frac{p}{r} \\ 0 & \text { otherwise }\end{cases}
$$

with $r=2^{\left\lfloor\log _{2}(l-1)\right\rfloor}$ and $s=\frac{p(l-1-r)}{r}+1$.

Then, the corresponding basis matrices can be expressed as $\overline{\boldsymbol{\Phi}}_{p q}[k, l]=\mathbf{h}_{p}^{l}[k]$. Substituting them in the result of Lemma 2 , a resolution-limited approximation of the original image is obtained from a set of its geometric moments. Fig. 6 shows the obtained results for the same pattern as in the previous examples and Table. 1 indicate the image size for which the reconstruction can be safely done.

When approximating an image, an often used strategy is to divide it into blocks. As a final example, let us consider the $256 \times 256$ image in Fig. 7 , upper left, which has been split down into $16 \times 16$ blocks. For each block, the geometric moments up to order $(4,4)$ have been computed. Fig. 7 also shows the reconstructed images using pseudoinverses applying Lemma 1, and Fourier and Haar basis using Lemma 2.

\section{Conclusions}

A desirable property for the basis functions used in the approximation of an image is that they concentrate most of the information in a reduced amount of coefficients. What information means depends on the interpretation of the basis; however, most common applications refer to bandwidth or spatial resolution, which are associated with Fourier and Haar coefficients, respectively. Then, setting a relationship between these coefficients and moments provides a straightforward interpretation of the information contained in moments, as well as 

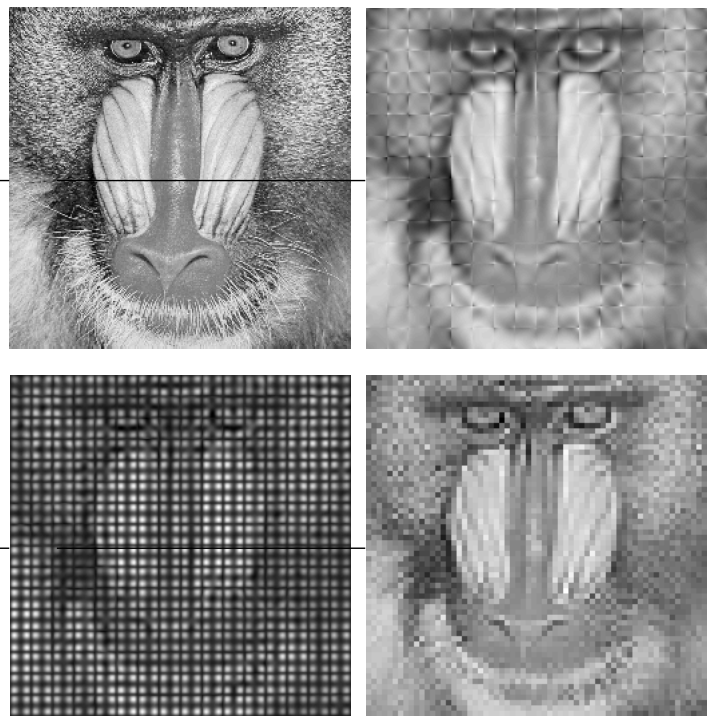

Figure 7: Clockwise from upper left: original image, and reconstructions using pseudoinverses, Haar and Fourier bases, respectively. Data: $256 \times 256$ image subdivided into $16 \times 16$ blocks; moments up to order $(4,4)$ used.

a method for reconstructing an image from a given set of moments. None of the former methods provided the proper setting to introduce these constraints.

Images are nonstationary two-dimensional signals with edges, textures, and deterministic objects at different locations. Nonstationary signals are, in general, characterized by their local features rather than their global ones. Nevertheless, we have recovered images by introducing constrains on either its spatial or frequency resolution, which are global constraints. If we want to use local constraints, we have to simultaneously introduce time and frequency constraints. In other words, we need a time-frequency joint representation, such as that obtained using a short-time Fourier transform or, in general, a wavelet transform. The possibilities are unlimited and the problem is to find a criterion for selecting a basis that is intrinsically well adapted to represent a class of images. By assuming a certain energy distribution on the timefrequency plane for the image to be recovered from its moments, one can choose or even build a proper basis that best represents the image with few coefficients. Actually, the algorithm presented in [3] can readily used here to find the best basis in families of wavelet packet bases or local cosine bases. This is a point that deserves further research.

The MATLAB implementation developed for the experiments reported in this paper can be downloaded from http://www-iri.upc.es/people/ porta.

\section{References}

[1] N.I. Akhiezer, The Classical Moment Problem and Some Related Questions in Analysis, Hafner Publishing Co., New York, 1965.

[2] B. Beckermann, "The Condition Number of Real Vandermonde, Krylov and Positive Definite Hankel Matrices," Numerische Mathematik, Vol. 85, pp. 553-577, 2000.

[3] R.R. Coifman and M.V. Wickerhauser, "Entropybased algorithms for best basis selection," IEEE Trans. on Information Theory, Vol. 38, No. 2, pp. 713-718, 1992.

[4] G.T. Herman, Image Reconstruction from Projections: The Fundamentals of Computerized Tomography, Academic Press Inc., New York, 1980.

[5] H.J. Landau, Moments in Mathematics, Short Course Lecture Notes, Vol. 37, Amer. Math. Soc., Providence, R.I., 1987.

[6] S.X. Liao and M. Pawlak, "On image analysis by moments," IEEE Trans. on Pattern Analysis and Machine Intelligence, Vol. 18, No. 3, pp. 254-266, 1996.

[7] M. Macon and A. Spitzbart, "Inverses of Vandermonde matrices," The American mathematical Monthly, Vol. 65, No. 2, pp. 95-100, 1958.

[8] J. Martínez, Accumulation Moments. Theory and Applications, Ph.D. thesis, Technical University of Catalonia, Spain, 1998.

[9] J. Martínez and F. Thomas, "Efficient Computation of Local Geometric Moments," IEEE Trans. on Image Processing, Vol. 11, No. 9, pp. 1102-1112, 2002.

[10] P. Milanfar, Geometric estimation and reconstruction from tomographic data, Ph.D. thesis, Massachusetts Institute of Technology, 1993. 
[11] P. Milanfar, W.C. Karl, A.S. Willsky, "A moment-based variational approach to tomographic reconstruction," IEEE Trans. on Image Processing, Vol. 5, No. 3, pp. 459-470, 1996.

[12] R. Mukundan, S.H. Ong, and P.A Lee, "Image Analysis by Tchebichef Moments," IEEE Trans. on Image Processing, Vol. 10, No. 9, pp. 13571364, 2001.

[13] T.B. Nguyen and B.J. Oommen, "MomentPreserving piecewise linear approximations of signals and images," IEEE Trans. on Pattern Analysis and Machine Intelligence, Vol. 19, No. 1, pp. 84-91, 1997.

[14] R.C. Papademetriou, "Reconstructing with moments," Proc. Int. Conf. on Pattern Recognition, pp. 476-480, 1992.

[15] M. Pawlak, "On the reconstruction aspects of moment descriptors," IEEE Trans. on Information Theory, Vol. 38, No. 6 pp. 1698-1708, 1992.

[16] J.A. Shohat and J.D. Tamarkin, The Problem of Moments, American Mathematical Society Surveys No. 1, 1943.

[17] M.R. Teague, "Image analysis via the general theory of moments," Journal of the Optical Society of America, Vol. 70, No. 8, pp. 920-930, 1980.

[18] D.V. Widder, "The Moment Problem," chapter 3 in The Laplace Transform, pp. 100-101, Princeton University Press, Princeton, NJ, 1941. 